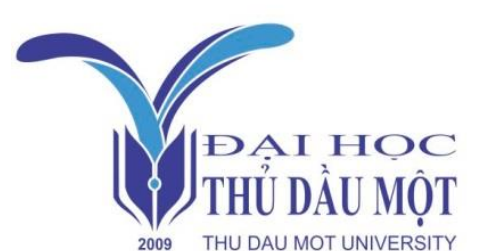

2009 THU DAU MOT UNIVERSITY

\section{Thu Dau Mot University Journal of Science}

journal homepage: ejs.tdmu.edu.vn

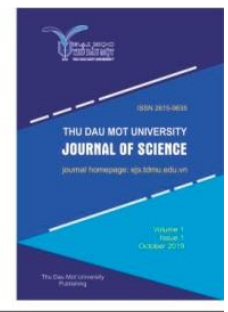

\title{
Simulating 3r robot dynamics using imported cad in Maplesim
}

by Nguyen Van Tan (Thu Dau Mot University)

Article Info: Received 20 Oct 2020, Accepted 25 Nov 2020, Available online 15 Dec, 2020

Corresponding author: tannv@tdmu.edu.vn

https://doi.org/10.37550/tdmu.EJS/2020.04.084

\begin{abstract}
With the development of information technology, many applications of robots are increasingly being applied to support research, learning, and teaching. This paper mainly investigates the modeling and simulation of a robotic arm with 3 degrees of freedom (dofs) for different applications. First, Kinematics and dynamics model of the robot based on the standard Denavit Hartenberg $(D-H)$ modeling method, where the forward kinematics of robot is analyzed and computed to obtain by using the inverse kinematics, and then the solution of the robot dynamics is derived. Second, a CAD model of the robot is designed on CATIA software to convert to MapleSim software to simulation and control. Final, numerical simulation is presented to display results. This work provides a potential basis for the realization of the robotic arm in the industrial, education, and research field, which is of great significance for improving manufacturing efficiency and support teaching and research in the robot field.
\end{abstract}

Keywords: Inverse kinematics, Forward Kinematics, MapleSim, Robot arm

\section{Introduction}

With the development of information and mechanical technologies, robots have been invented and applied to the manufacturing process, which can improve the production efficiency, enhance productivity, improve the working conditions and accelerate the industrial automation [1-2]. Several other applications of robot arms were used in the 
automotive industry and are often used for welding, painting, loading, and unloading [3] as well as in the medical field $[4,5]$. The robotic arm extends and expands the functions of the hand, foot, and brain. It can replace people's work in harsh environments such as danger, harmfulness, toxic, low temperature, and high heat: instead of people doing heavy and monotonous repetitive work, which can improve labour productivity and ensure product quality. Its application has been extended to the field of space exploration, deep-sea development, nuclear science research and medical welfare. A robot arm is a new technology emerging in the field of modern automatic control and has become an important part of modern industrial systems [6]. The robot arm consists of the robot arm, controller, servo drive system, detection and sensing components. It is a type of automated production equipment with features such as human-like operation, automatic control, reprogrammable, and can complete various operations in threedimensional space.

To support robot research, learning, and research, many applications software support the design, calculation, analysis, simulation, and optimization of the robot control process has been rapidly developing in it. Some software is used a lot such as Simmechanics to simulation robot arm which was shown in [7, 8], and MSC Adams to analysis and simulation robot arm that was also shown in [9, 10], then, MapleSim has used analysis, dynamics simulation and optimization shown in [11-13]. Particularly, with LMS AmeSim, Simcenter, and MSC Adams, the simulation results are treated as real results that can be accepted in $[14,15]$.

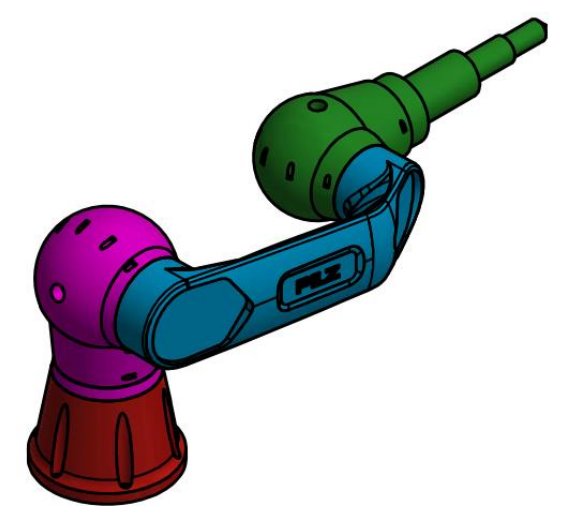

Figure 1. The robot arm model $3 \mathrm{R}$

In this paper, An application study of the Maplesim software to control and simulate a 3R robot arm. First, the robot arm model is built in CAD software such as Solidwork or Inventor, or Catia that is shown in Figure 1. From this, this model is imported to MapleSim. And then, establish constraints, the relationships of robot arm components 
as well as DC motor actuators to control the robot arm. Here, PID controllers are also utilized in the control process.

\section{Denavit and Hartenbergh Representation}

The kinematic equations use matrix algebra to build constrain functions between the joint variables and the world coordinate location of the end-effector (position and orientation) based on the spatial configuration of a particular robot arm. A systematic and generalized approach to represent the kinematic equations of a serial link robot arm was proposed by Denavit \& Hartenbergh (D-H).

The notation will be explained on a sample robot arm which is illustrated in figure 2 . The robot system has three degrees of freedom, based on the transformation matrix Ai (i $=1,2,3$ ) described by the D-H notation, we can get the pose of the robot's end-effector only given the individual joint variables and the rod length parameters of the robot [7].

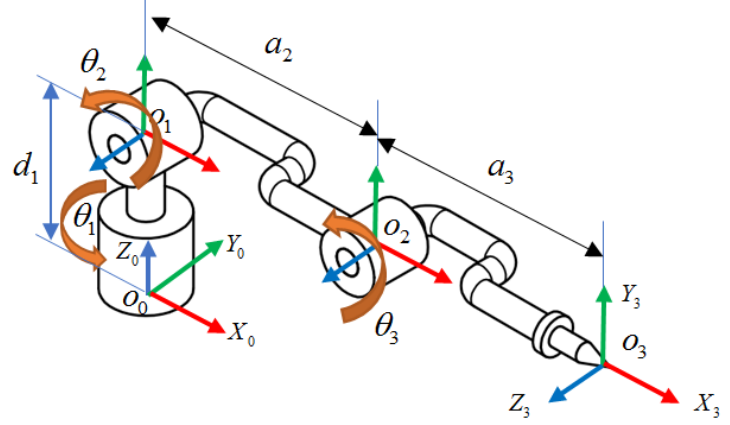

Figure 2. Coordinate system of robot

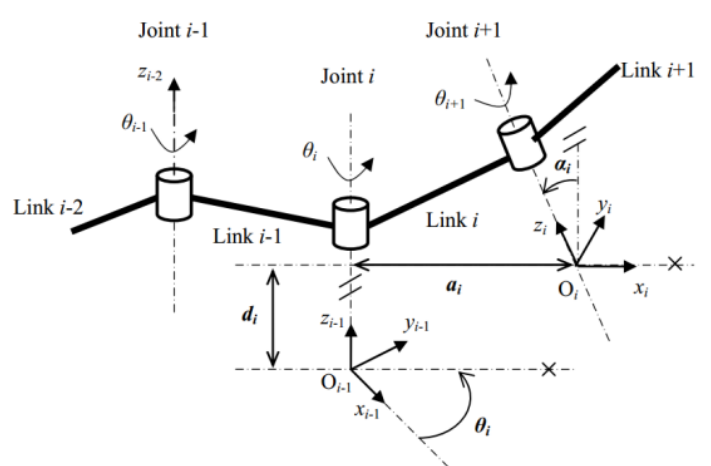

Figure 3. Structure kinematic parameters for the general link $i^{\text {th }}$

The homogenous coordinate transformation matrix ${ }^{i} T_{i-1}$ can be written as

$$
{ }^{i} T_{i-1}=\left[\begin{array}{cccc}
C_{\theta_{i}} & -S_{\theta_{i}} C_{\alpha_{i}} & S_{\theta_{i}} S_{\alpha_{i}} & a_{i} C_{\theta_{i}} \\
S_{\theta_{i}} & C_{\alpha_{i}} C_{\theta_{i}} & -S_{\alpha_{i}} C_{\theta_{i}} & a_{i} S_{\theta_{i}} \\
0 & S_{\alpha_{i}} & C_{\alpha_{i}} & d_{i} \\
0 & 0 & 0 & 1
\end{array}\right]
$$

where

$$
C_{\theta_{i}}=\cos \theta_{i} ; S_{\theta_{i}}=\sin \theta_{i} ; C_{\alpha_{i}}=\cos \alpha_{i} ; S_{\alpha_{i}}=\sin \alpha_{i}
$$

and $a_{i}, d_{i}, \alpha_{i}, \theta_{i}$ are the geometric parameters of the relative ith joint as shown in Fig 3 . 
Nguyen van Tan- Volume 2- Issue 4-2020, p.

They can be defined as:

$\alpha_{i}$ is the angle between $z_{i-1}$ and zi about $x_{i}$

$d_{i}$ is the distance between origin $\mathrm{o}_{\mathrm{i}-1}$ and the intersection of the $z_{i-1}$ axis with the $x_{i}$ axis along $z_{i-1}$

$\theta_{i}$ is the angle between $x_{i-1}$ and $x_{i}$ about $z_{i-1}$.

$a_{i}$ is the distance between origin Oi and the intersection of the $z_{i-1}$ axis with the $x_{i}$ axis along $x_{i}$

If a position vector $P_{i}$ is given in the $i^{\text {th }}$ coordinate frame, then it can be expressed in the $(i-1)^{\text {th }}$ coordinate system as the vector $P_{i-1}$ by

$$
p_{i-1}={ }^{i} T_{i-1} p_{i}
$$

The homogenous transformation matrix from the $\mathrm{n}^{\text {th }}$ coordinate frame to the base coordinate frame can be determined by multiplying ${ }^{i} T_{i-1}(\mathrm{i}=1,2, \ldots, \mathrm{n})$ together in sequence, such as

$$
{ }^{n} T_{0}={ }^{1} T_{0}^{2} T_{1} \ldots{ }^{n} T_{n-1}
$$

Therefore, a position vector $p_{n}$ of the $n^{\text {th }}$ coordinate frame can be determined for the base coordinate system as:

$$
{ }^{o} p_{n}={ }^{n} T_{0}^{-1} p_{0}
$$

Using the D-H procedure, the coordinate frames are defined and the structural geometric parameters of the three-link robot arm are presented in Table 1.

TABLE 1. Joint-Link Parameters for 3R Robot arm

\begin{tabular}{|c|c|c|c|c|}
\hline Link & $a_{i}$ & $\alpha_{i}$ & $d_{i}$ & $\theta_{i}$ \\
\hline 1 & 0 & $90^{0}$ & $d_{1}$ & $\theta_{1}$ \\
\hline 2 & $a_{2}$ & 0 & 0 & $\theta_{2}$ \\
\hline 3 & $a_{3}$ & 0 & 0 & $\theta_{3}$ \\
\hline
\end{tabular}

Therefore, the coordinate transformation matrices ${ }^{i} T_{i-1}(i=1,2,3)$ relating the coordinates of the $\boldsymbol{i}^{\text {th }}$ frame to those of the $(i-1)^{\text {th }}$ frame can be written using equation (1.1) as follows 


$$
\begin{aligned}
{ }^{1} T_{0} & =\left[\begin{array}{cccc}
C_{\theta_{1}} & 0 & S_{\theta_{i}} & 0 \\
S_{\theta_{1}} & 0 & -C_{\theta_{i}} & 0 \\
0 & 1 & 0 & d_{i} \\
0 & 0 & 0 & 1
\end{array}\right] \\
{ }^{2} T_{1} & =\left[\begin{array}{cccc}
C_{\theta_{2}} & -S_{\theta_{2}} & 0 & a_{2} C_{\theta_{2}} \\
S_{\theta_{2}} & C_{\theta_{2}} & 0 & a_{2} S_{\theta_{2}} \\
0 & 0 & 1 & 0 \\
0 & 0 & 0 & 1
\end{array}\right] \\
{ }^{3} T_{2} & =\left[\begin{array}{cccc}
C_{\theta_{3}} & -S_{\theta_{3}} & 0 & a_{3} C_{\theta_{3}} \\
S_{\theta_{3}} & C_{\theta_{3}} & 0 & a_{3} S_{\theta_{3}} \\
0 & 0 & 1 & 0 \\
0 & 0 & 0 & 1
\end{array}\right]
\end{aligned}
$$

The homogenous transformation matrix ${ }^{3} T_{0}$ from the coordinate frame attached to the end-effector to the base coordinate frame can be represented as

$$
\begin{aligned}
{ }^{3} T_{0} & ={ }^{1} T_{0}^{2} T_{1}^{3} T_{2} \\
& =\left[\begin{array}{cccc}
C_{\theta_{1}} C_{\theta_{23}} & -C_{\theta_{1}} S_{\theta_{23}} & S_{\theta_{1}} & C_{\theta_{1}}\left(a_{3} C_{\theta_{23}}+a_{2} C_{\theta_{2}}\right) \\
S_{\theta_{1}} C_{\theta_{23}} & -S_{\theta_{1}} S_{\theta_{23}} & -C_{\theta_{1}} & S_{\theta_{1}}\left(a_{3} C_{\theta_{23}}+a_{2} C_{\theta_{2}}\right) \\
S_{\theta_{23}} & C_{\theta_{23}} & 0 & a_{3} S_{\theta_{23}}+a_{2} S_{\theta_{2}}+d_{1} \\
0 & 0 & 0 & 1
\end{array}\right]
\end{aligned}
$$

Where $C_{\theta_{23}}=\cos \left(\theta_{2}+\theta_{3}\right) ; S_{\theta_{23}}=\sin \left(\theta_{2}+\theta_{3}\right)$;

Based on this transformation matrix, the forward kinematics can determine a precise coordinates of the robot arm in the workspace according to the value of each joint angle variable.

The geometric approach is applied to find the inverse kinematics of the three-link robot arm shown in Fig 4. A point $\mathrm{P}$ representing a given position of the end-effector, which is the origin of the last coordinate frame concerning the base coordinate system, can be expressed by

$$
p=\left[\begin{array}{lll}
p_{x} & p_{y} & p_{z}
\end{array}\right]^{T}
$$


Nguyen van Tan- Volume 2- Issue 4-2020, p.

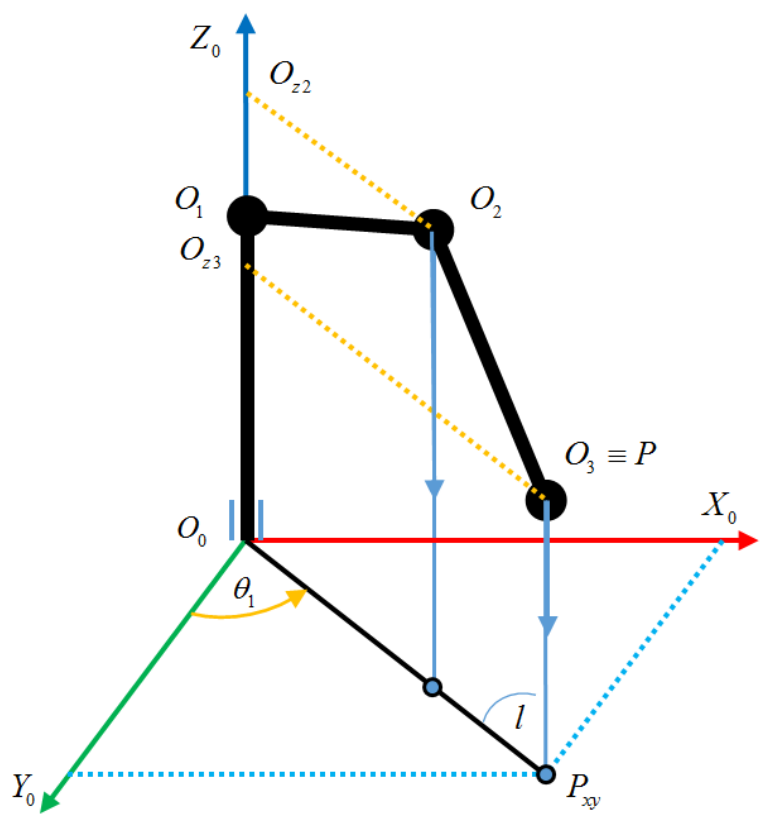

Figure 4. Describe the $\mathrm{P}$ position in the original coordinate system

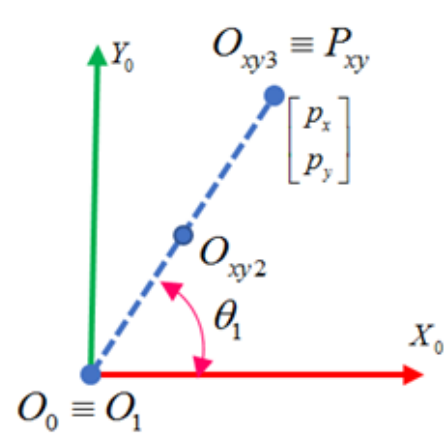

a) P position in $X_{0} Y_{0}$ plan

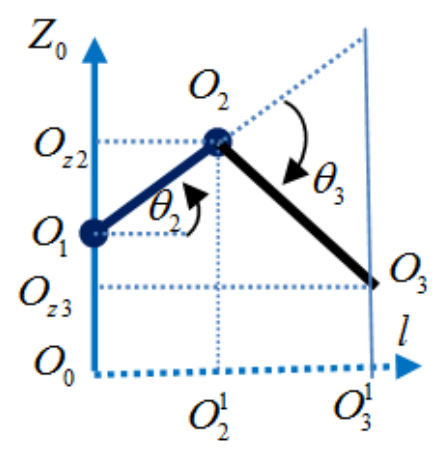

b) $P$ position in l plan

Figure 5. Describe the $\mathrm{P}$ position in the $2 \mathrm{D}$ coordinate system (where $l$ is the plan of $\left(z_{0}, P\right)$ ) From (1.8) and (1.9), we have

$$
p=\left[\begin{array}{c}
p_{x} \\
p_{y} \\
p_{z}
\end{array}\right]=\left[\begin{array}{c}
C_{\theta_{1}}\left(a_{3} C_{\theta_{23}}+a_{2} C_{\theta_{2}}\right) \\
S_{\theta_{1}}\left(a_{3} C_{\theta_{23}}+a_{2} C_{\theta_{2}}\right) \\
a_{3} S_{\theta_{23}}+a_{2} S_{\theta_{2}}+d_{1}
\end{array}\right]
$$

From Fig 4 and Fig 5, we have

$$
\begin{gathered}
O_{0} P_{x y}=\sqrt{p_{x}^{2}+p_{y}^{2}} \\
P_{x}=O_{0} P_{x y} \cos \theta_{1} \\
P_{y}=O_{0} P_{x y} \sin \theta_{1}
\end{gathered}
$$


Therefore, $\theta_{1}$ variable can be calculated as

$$
\theta_{1}=\tan ^{-1}\left(\frac{p_{y}}{p_{x}}\right)
$$

Moreover, in Fig. 5. We have

$$
\begin{gathered}
O_{0} O_{3}^{1}=O_{0} O_{2}^{1}+O_{2}^{1} O_{3}^{1}=a_{2} \cos \theta_{2}+a_{3} \cos \left(\theta_{2}+\theta_{3}\right) \\
O_{0} O_{z 3}=O_{0} O_{1}+O_{1} O_{z 2}-O_{1} O_{z 3} \\
=d_{1}+a_{2} \sin \theta_{2}-a_{3} \sin \left(\theta_{2}+\theta_{3}\right)
\end{gathered}
$$

where

$$
O_{0} O_{3}^{1}=O_{0} P_{x y}=\sqrt{p_{x}^{2}+p_{y}^{2}} ; \quad O_{0} O_{z 3}=p_{z}
$$

Solve equations (1.15) and (1.16) we have

$$
\begin{gathered}
\cos \theta_{3}=\frac{p_{x}^{2}+p_{y}^{2}+\left(p_{z}-d_{1}\right)^{2}-a_{2}^{2}-a_{3}^{2}}{2 a_{2} a_{3}}=q \\
\sin \theta_{3}= \pm \sqrt{(1-q)^{2}}
\end{gathered}
$$

Therefore, $\theta_{3}$ can finally be calculated as

$$
\theta_{3}=\tan ^{-1}\left[ \pm \frac{\sqrt{(1-q)^{2}}}{q}\right]
$$

and $\theta_{2}$ can be calculated as

$$
\theta_{2}=\tan ^{-1}\left[ \pm \frac{\sqrt{(1-\delta)^{2}}}{\delta}\right]-\varphi-\theta_{3}
$$

where

$$
\varphi=\tan ^{-1}\left[ \pm \frac{\sqrt{p_{x}^{2}+p_{y}^{2}}}{p_{z}-d_{1}}\right] ; \delta=\frac{p_{x}^{2}+p_{y}^{2}+\left(p_{z}-d_{1}\right)^{2}+a_{3}^{2}-a_{2}^{2}}{2 a_{3} \sqrt{p_{x}^{2}+p_{y}^{2}+\left(p_{z}-d_{1}\right)^{2}}}
$$

\section{Import Cad Robot Model To MapleSim}

MapleSim software was used for the dynamic analysis of the robot model. The basic prerequisites includes geometric correctness and the validity of constraints were done in Catia software. The material of the robot parts (as this determines the mass and other basic dynamic parameters) is equally significant as observed during reverse engineering (RE). Once this is done in CATIA the "Motion Study" itself is capable of exporting these details along with the geometric model to MapleSim. Verification of the coherence in inertial parameters can be confirmed in both MapleSim and CATIA platforms 
Nguyen van Tan- Volume 2- Issue 4-2020, p.

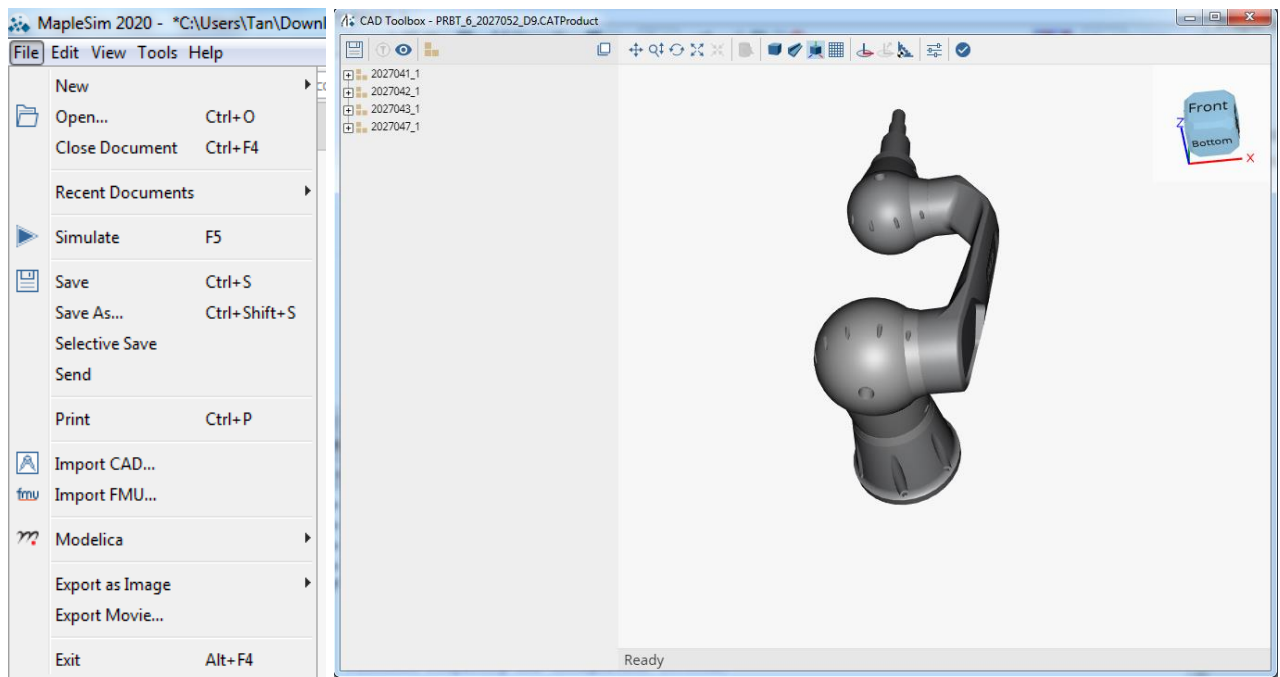

Figure 6. Robot arm model in MapleSim

To import CAD to Maplesim, from the menu, you choose a file then select Import CAD as shown in figure 6 . Inertial parameters of the robot parts are calculated automatically as shown in figure 7.

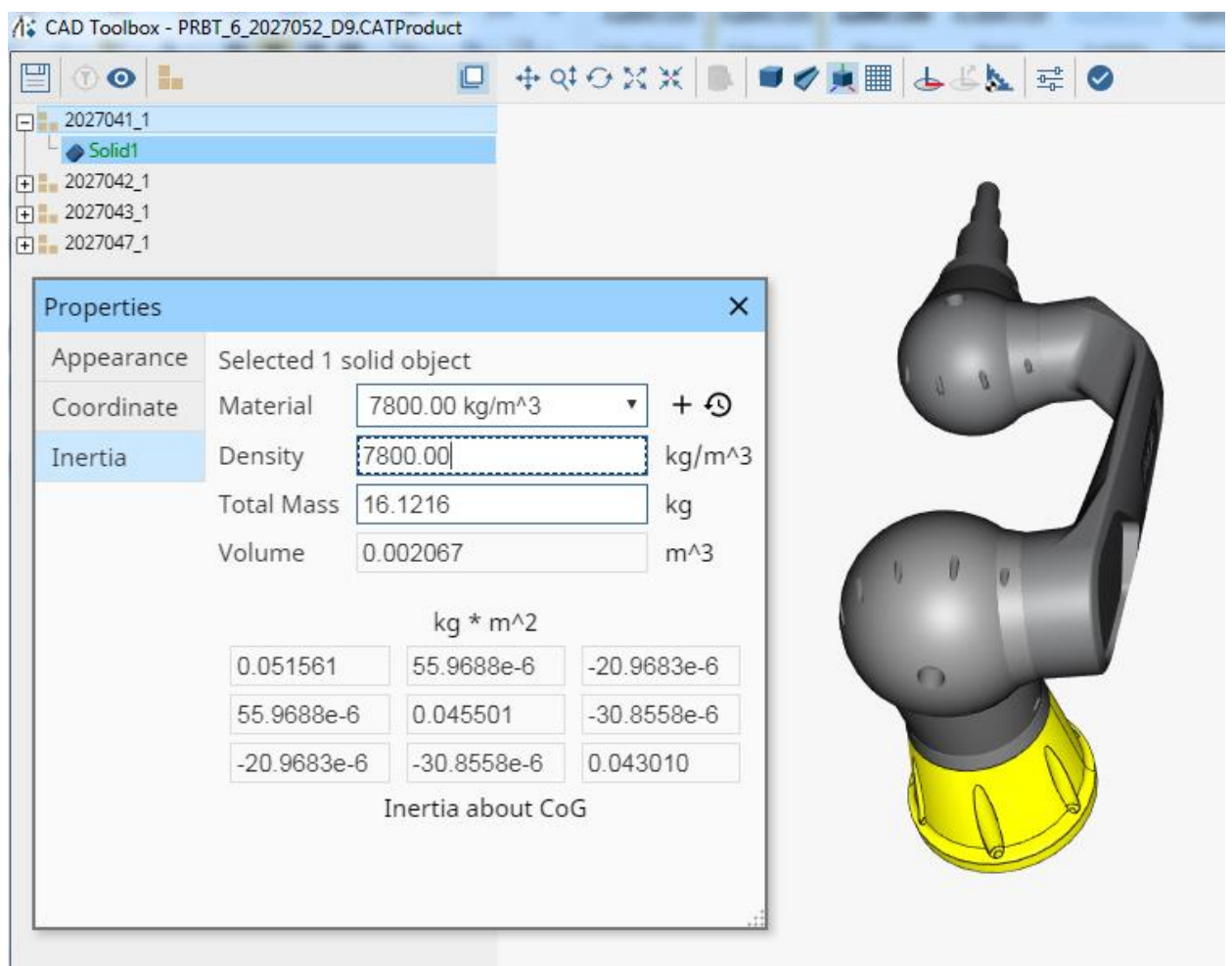

Figure 7. The scheme describes the inertial mass of the base plate of the robot

Then, the Robot model is converted to MapleSim software and implement connection components with joints and actuators to perform the control process as shown in figure 8 . 


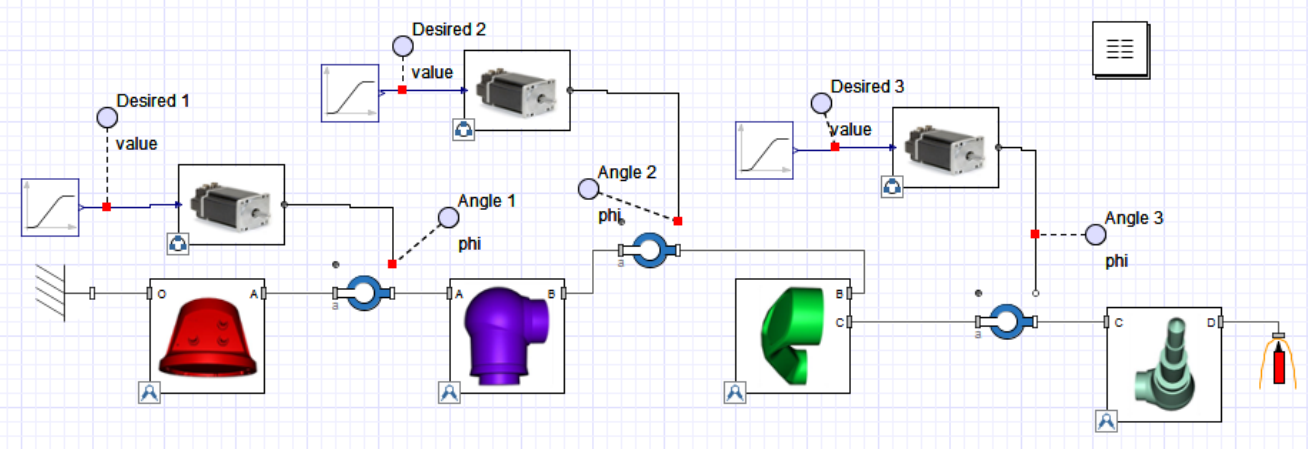

Figure 8. Scheme of the robot arm in Maplesim to control

Three actuators are used to control the robot by three DC motors shown in figure. 9 where the DC motors are controlled by three PID controllers

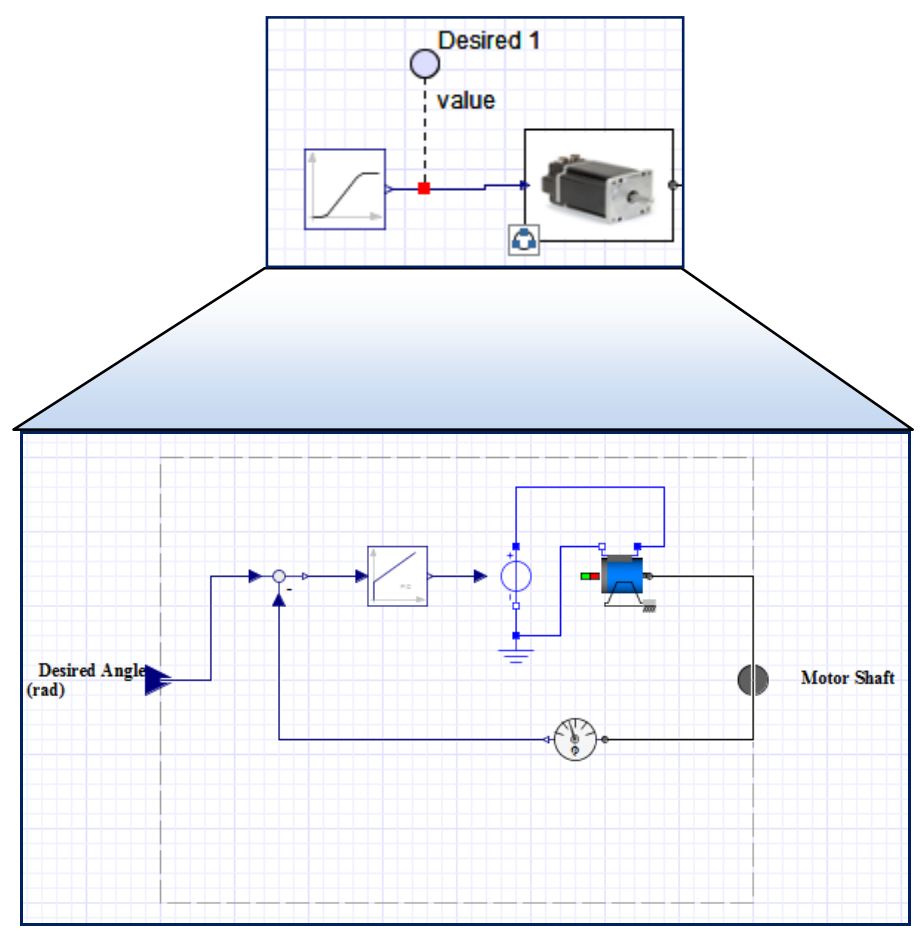

Figure 9. Control Diagram of DC motor with PID controller

\section{Simulation Results}

By experience to choose parameters of PID controllers as:

$$
\begin{gathered}
\mathrm{Kp}_{1}=15 ; \mathrm{Ki}_{1}=5, \mathrm{Kd}_{1}=2 ; \\
\mathrm{Kp}_{2}=30 ; \mathrm{Ki}_{2}=5, \mathrm{Kd}_{2}=10 ; \\
\mathrm{Kp}_{3}=50 ; \mathrm{Ki}_{3}=8, \mathrm{Kd}_{3}=2 ;
\end{gathered}
$$


Nguyen van Tan- Volume 2- Issue 4-2020, p.

The response result of the first joint is shown in figure 10
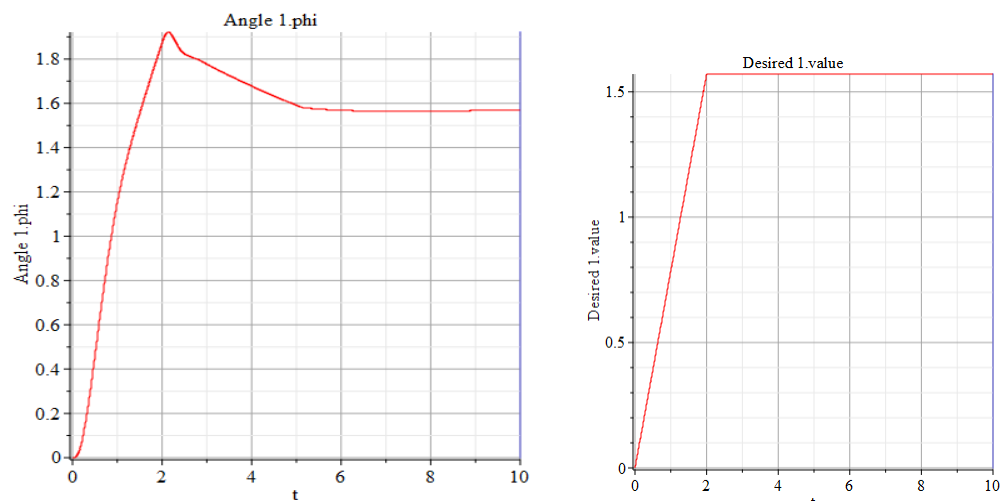

Figure 10. Response angle and desired angle of the first joint

The response result of the second joint is shown in figure 11
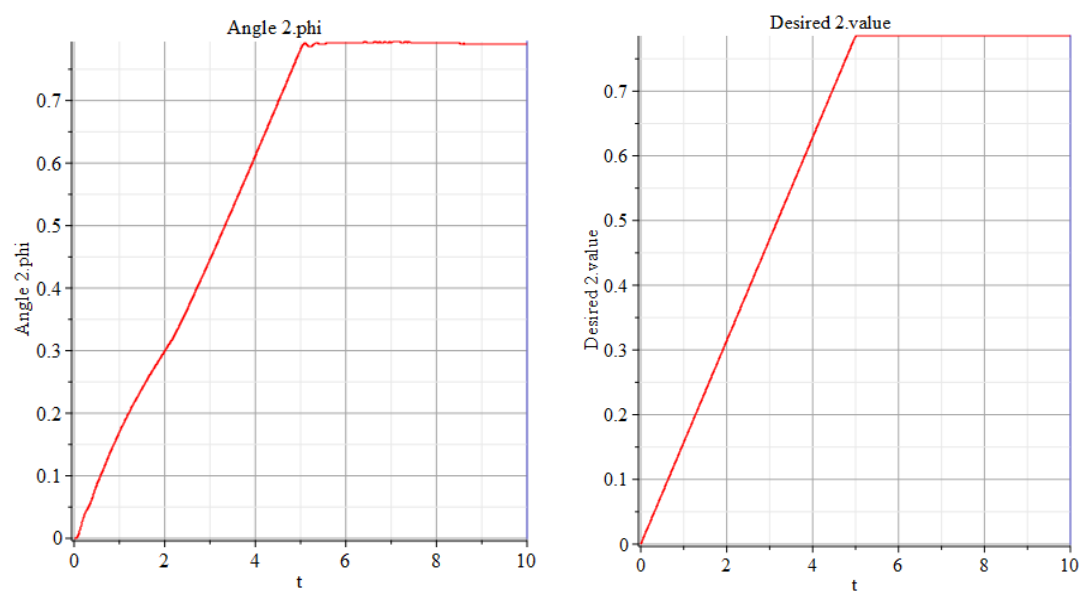

Figure 11. Response angle and the desired angle of the second joint The response result of the third joint is shown in figure 12
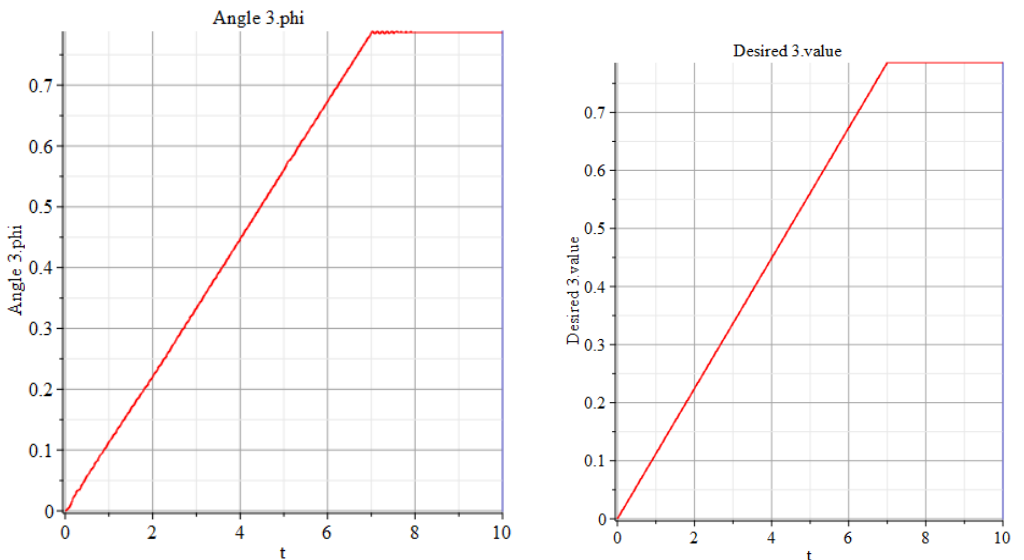

Figure 12. Response angle and desired angle of the third joint 
The simulation result of the robot arm is shown in figure 13.

3D simulation robot result is stimulated when given three angles of three axes shown in figure 10 , figure. 11 , and figure 12 . The trajectory of the end-effector is displayed by the red trajectory as shown in figure 13.

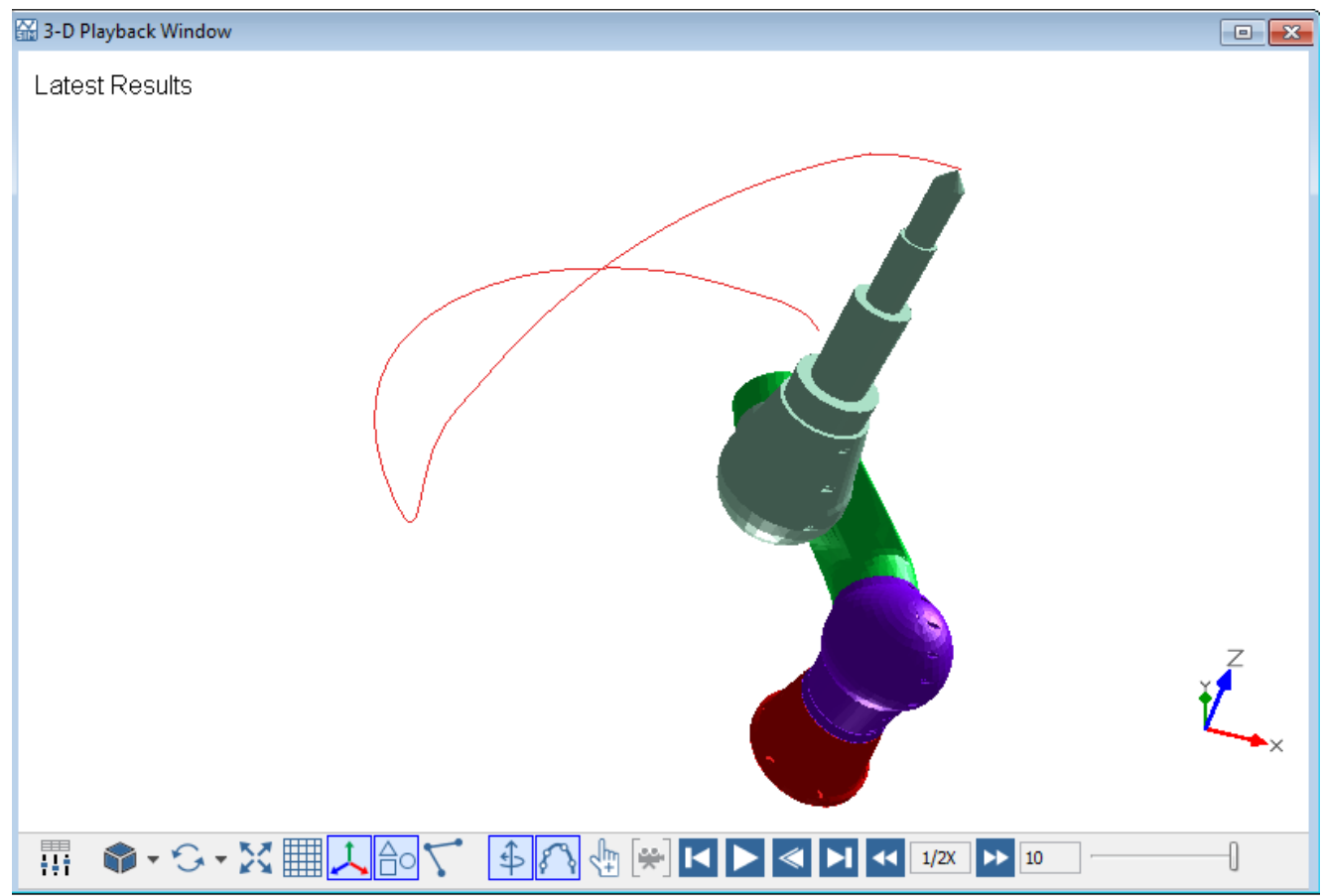

Figure 13. The simulation result of the robot in 3D Maplesim

\section{Conclusion}

The rotation angles of the robot arm joints are calculated based on inverse kinematics of the D-H convention. The robot is designed in CATIA to easy converted to Maplesim software. The robot arm is set up in maplesim to control and simulation. Simulation results were obtained as expected under using PID controller that shown in the figures from figure 10 to figure 13 .

Adknowledge: This Research is support by Truong Dai hoc Thu Dau Mot, Binh Duong province, Viet Nam

\section{References}


[1] Y. He et al., (2019). Dynamic Modeling, Simulation, and Experimental Verification of a Wafer Handling SCARA Robot With Decoupling Servo Control. IEEE Access, vol. 7, pp. 47143-47153, doi: 10.1109/ACCESS.2019.2909657.

[2] Taylan Das M, Canan Dulger L., (2005). Mathematical Modelling, simulation and experimental verification of a SCARA Robot. Simulation Modelling Practice and Theory, Vol 13, pp. 257-271;

[3] W. Jin, et al., (2020). Wire Arc Additive Manufacturing of Stainless Steels: A Review. Appl. Sci. , 10(5), 1563; https://doi.org/10.3390/app10051563

[4] M. Wei, Q. Liu, and, et al., (2015). Recent development of mechanisms and control strategies for robot-assisted lower limb rehabilitation. Mechatronics 31, 132-145

[5] S. S. KAMLESH, A. KUMAR, and et al., (2016). Review on Application of Robot Arm in Medical Field with Special Forcus on Image Guided Biopsies. International Journal of Mechanical And Production Engineering, 4(11),

[6] Toz, Met in, and Serdar Kucuk (2010). Dynamics simulation toolbox for industrial robot robot arms. Computer Applications in Engineering Education, 18(2), 319-330.

[7] J. Liu, Q. Luo, (2019). Modeling and Simulation of Robotic Arm in MATLAB for Industrial Applications. 11th International Conference on Intelligent Human-Machine Systems and Cybernetics (IHMSC), 978-1-7281-1859IEEE DOI 10.1109/IHMSC.2019.00086

[8] J. ZATOPEK, and et al., (2018). Dynamic simulation of the CAD model in SimMechanics with multiple uses. Turkish Journal of Electrical Engineering \& Computer Sciences, urk J Elec Eng \& Comp Sci ., 6: 1278 \{ 1290

[9] T, Amit., "Forward Kinematic Analysis, Simulation \& Workspace Tracing of Anthropomorphic Robot Robot arm By Using MSC. ADAMS". International Journal of Innovative Research in Science, Engineering and Technology. 04. Doi: 10.15680/IJIRSET.2015.0401009.

[10] Ratiu, Mariana, and et al., (2018). Modeling in ADAMS of a 6R industrial robot. MATEC Web of Conferences. Doi: 10.1051/matecconf/201818402006.

[11] J. Hřebíček, and et al., "Modelling with Maple and MapleSim". Proceedings 22nd European Conference on Modelling and Simulation CECMS Loucas S. Louca, Yiorgos Chrysanthou, Zuzana Oplatková, Khalid Al-Begain (Editors) ISBN: 978-0-9553018-5-8 / ISBN: 978-0-9553018-6-5 (CD)

[12] D. Wright and O. Soroka (2012). Modeling and simulation of a linear piezoelectric stepper motor in maplesim. IEEE International Ultrasonics Symposium, Dresden, pp. 2746-2749, doi: 10.1109/ULTSYM.2012.0688.

[13] Korobkine, A., Boimer, R., Pantelic, V., Shah, S. et al., (2018). Automatic Calibrations Generation for Powertrain Controllers Using MapleSim. SAE Technical Paper https://doi.org/10.4271/2018-01-1458.

[14] P, Aron, and et al., (2015). Real-time co-simulation platform for electromechanical vehicle applications). 9th International Symposium on Advanced Topics in Electrical Engineering, ATEE (2015). 240-243. Doi:10.1109/ATEE.2015.7133772.

[15] X. Feixiang and et al., (2018). Modeling and co-simulation based on Adams and AMESim of pivot steering system. The Journal of Engineering. Doi: 10.1049/joe.2018.9018. 
Thu Dau Mot University Journal of Science - Volume 2 - Issue 4-2020 